\title{
New Diagnostic Approach for Esophageal Squamous Cell Neoplasms Using Linked Color Imaging and Blue Laser Imaging Combined with Iodine Staining
}

\author{
Masato Tsunoda' ${ }^{1}$ Yoshimasa Miura ${ }^{1}$, Hiroyuki Osawa ${ }^{1}$, Tsevelnorov Khurelbaatar', Mio Sakaguchi ${ }^{2}$, Hisashi Fukuda', \\ Alan Kawarai Lefor ${ }^{3}$ and Hironori Yamamoto ${ }^{1}$ \\ ${ }^{1}$ Division of Gastroenterology, Department of Medicine, ${ }^{2}$ Department of Diagnostic Pathology, ${ }^{3}$ Department of Surgery, Jichi Medical \\ University, Shimotsuke, Japan
}

A 62-year-old man with a flat early esophageal cancer was referred for endoscopic treatment. White light imaging revealed a pale red lesion, whereas linked color imaging (LCI) and blue laser imaging (BLI) yielded purple and brown images, respectively. Iodine staining demonstrated a large unstained area with a homogenous but very weak pink-color sign. This area appeared more clearly as purple and green on LCI and BLI, respectively; however, a different colored portion was observed at the 4 o'clock position inside the iodineunstained area. Histopathology findings of the resected specimen revealed squamous intraepithelial neoplasia at the 4 o'clock position and an esophageal squamous cell carcinoma in the remaining iodine-unstained area. LCI and BLI combined with iodine staining produce characteristic images that overcomes the pink-color sign, reflecting the histological features of a flat esophageal neoplasm. This new method is useful for detailed evaluation of early flat squamous cell neoplasms. Clin Endosc 2019;52:497-501

Key Words: Early esophageal cancer; Image enhanced endoscopy; Linked color imaging; Blue laser imaging; Iodine staining

\section{INTRODUCTION}

Endoscopy enables diagnosis of neoplastic lesions at an early stage, which may be amenable to endoscopic treatment to preserve the organ. ${ }^{1}$ Early esophageal neoplasms pose a diagnostic challenge using white light imaging (WLI) and image-enhanced endoscopy including narrow band imaging $(\mathrm{NBI})^{2-5}$ and blue laser imaging (BLI).$^{6-8}$ WLI combined with iodine staining has been routinely performed as unstained areas are visualized as a pink-color sign in presence of malignancy. ${ }^{9}$ Occasionally, differentiation of malignant lesions to

Received: October 29, 2018 Revised: December 21, 2018 Accepted: March 18, 2019

Correspondence: Hiroyuki Osawa

Division of Gastroenterology, Department of Medicine, Jichi Medical University, 3311-1 Yakushiji, Shimotsuke, Tochigi 329-0498, Japan

Tel: +81-285-58-7539, Fax: +81-285-44-0047, E-mail: osawa@jichi.ac.jp

ORCID: https://orcid.org/0000-0002-1479-0475

(c) This is an Open Access article distributed under the terms of the Creative Commons Attribution Non-Commercial License (http://creativecommons.org/ licenses/by-nc/3.0) which permits unrestricted non-commercial use, distribution, and reproduction in any medium, provided the original work is properly cited. be treated from other unstained lesions is difficult using these diagnostic modalities. ${ }^{10}$

The current study attempted to demonstrate the lesion in a patient with an early flat esophageal neoplasm using linked color imaging (LCI) and BLI combined with iodine staining. We present a new finding that LCI and BLI combined with iodine staining can produce characteristic images that overcome the pink-color sign and reflect in detail the histological features of a flat esophageal neoplasm.

\section{CASE REPORT}

A 62-year-old man with a flat type early esophageal cancer was referred for endoscopic submucosal dissection. Hypopharyngeal carcinoma was synchronously diagnosed by using upper gastrointestinal endoscopy by the referring physician. History of the patient revealed a habit of smoking (10 cigarettes/day for 42 years) and drinking alcohol $(700 \mathrm{~mL}$ beer/day). Physical examination revealed a mass in the right 
neck caused by the hypopharyngeal carcinoma and no other abnormal findings. Baseline laboratory investigations were within normal limits. A computed tomography scan from the neck to the pelvis showed a tumor in the right hypopharynx, but no metastatic lesions.

A 50-mm superficial esophageal cancer (0-IIb) was revealed in the distal esophagus using a laser endoscopic system (EGL590ZW, LL-7000 and VP-7000; Fujifilm Co., Tokyo, Japan). WLI revealed a pale red lesion, seen as purple on LCI and brown on BLI (Fig. 1). Magnified BLI revealed abnormal intrapapillary capillary loops (type B1). ${ }^{11}$ After staining with iodine solution, a large iodine-unstained area with an irregular shape was seen and the pink-color sign was positive but very weak under WLI. These areas remained purple on LCI and changed to green on BLI. The important issue is that the entire lesion was uniformly positive for the pink-color sign on WLI, whereas interestingly, a pale yellow area on LCI and brown area on BLI were found at the 4 o'clock position inside the iodine-unstained lesion, suggesting different histological findings (Fig. 1). These areas were evaluated by calculating the difference in color using the method of the Commission Internationale de l'Eclairage (CIE), as reported previously ${ }^{12}$ and by comparing with the histological findings of the resected specimens. Using this method, the difference was expressed as the distance between two locations corresponding to two colors in the CIE color space, which define a three-dimensional space $\left(L^{*}, a^{*}\right.$ and $\left.b^{*}\right)$ as a sphere. Greater distance implies high contrast with a high color difference. This was performed using commercially available image analysis software (Adobe Photoshop; Adobe Systems, San Jose, CA, USA). The color difference between the two regions was 28.9 on LCI and 26.6 on BLI both of which were higher than the 5.8 observed on WLI. Subsequently, markings were placed 2 or $3 \mathrm{~mm}$ outside the demarcated area and the lesion was resected using endoscopic submucosal dissection. Histopathologic findings of the resected specimen did not show esophageal squamous cell carcinoma but rather squamous intraepithelial neoplasia at the 4 o'clock position, and showed squamous cell carcinoma in the remaining iodine-unstained area (Fig. 2).

\section{DISCUSSION}

In this patient, LCI and BLI combined with iodine staining produced characteristic images that eliminated the pink-color sign seen on WLI and reflected the histological features of the flat esophageal neoplasm. Purple and green mucosa after iodine staining was readily identifiable, compared to the pink-color sign. In addition, this new modality could differentiate squamous cell carcinoma from squamous intraepithelial neoplasm by the difference in color. To the best of our knowledge, this is the first report of endoscopic findings using LCI
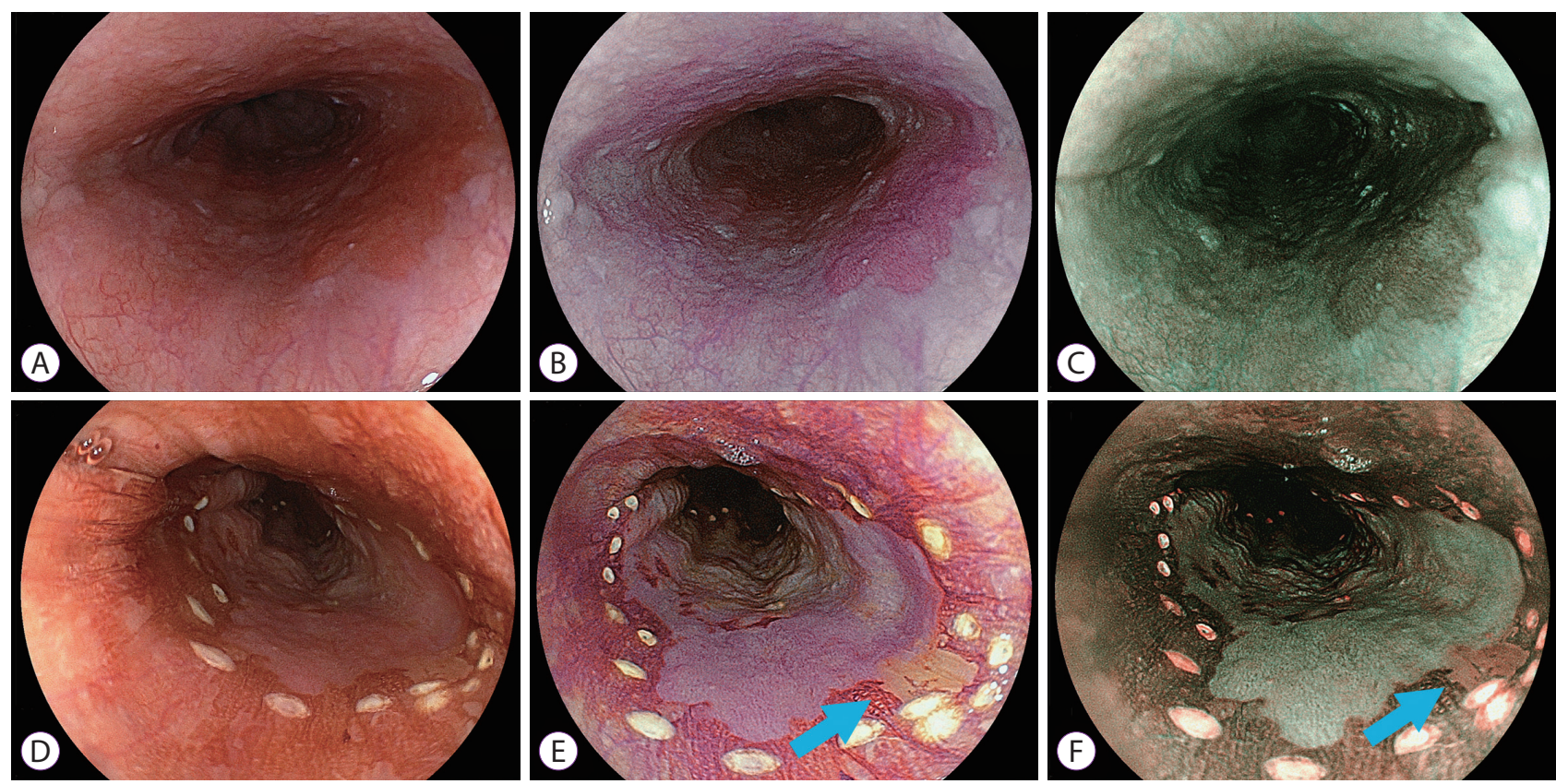

Fig. 1. Endoscopic images of esophageal squamous cell neoplasm. (A-C) Before iodine staining. (A) White light imaging shows slightly red lesion from the 3 to 5 o'clock position. (B) Linked color imaging shows a purple lesion. (C) Blue laser imaging shows a brown lesion. (D-F) Three minutes 35 seconds after iodine staining. (D) The entire lesion has a positive pink-color sign on white light imaging. (E) Linked color imaging shows a purple area in most of the main lesion but pale yellow mucosa at the 4 o'clock position (light blue arrow). (F) Blue laser imaging shows a green lesion in most of the main lesion but there is brown mucosa at the 4 o'clock position (light blue arrow). 
and BLI that provides new insights into the accurate diagnosis of esophageal squamous cell carcinoma.

Several endoscopic modalities have been developed to screen for early esophageal cancers such as WLI combined with iodine staining and image enhanced endoscopy. The prevalence of esophageal cancer is increasing worldwide.
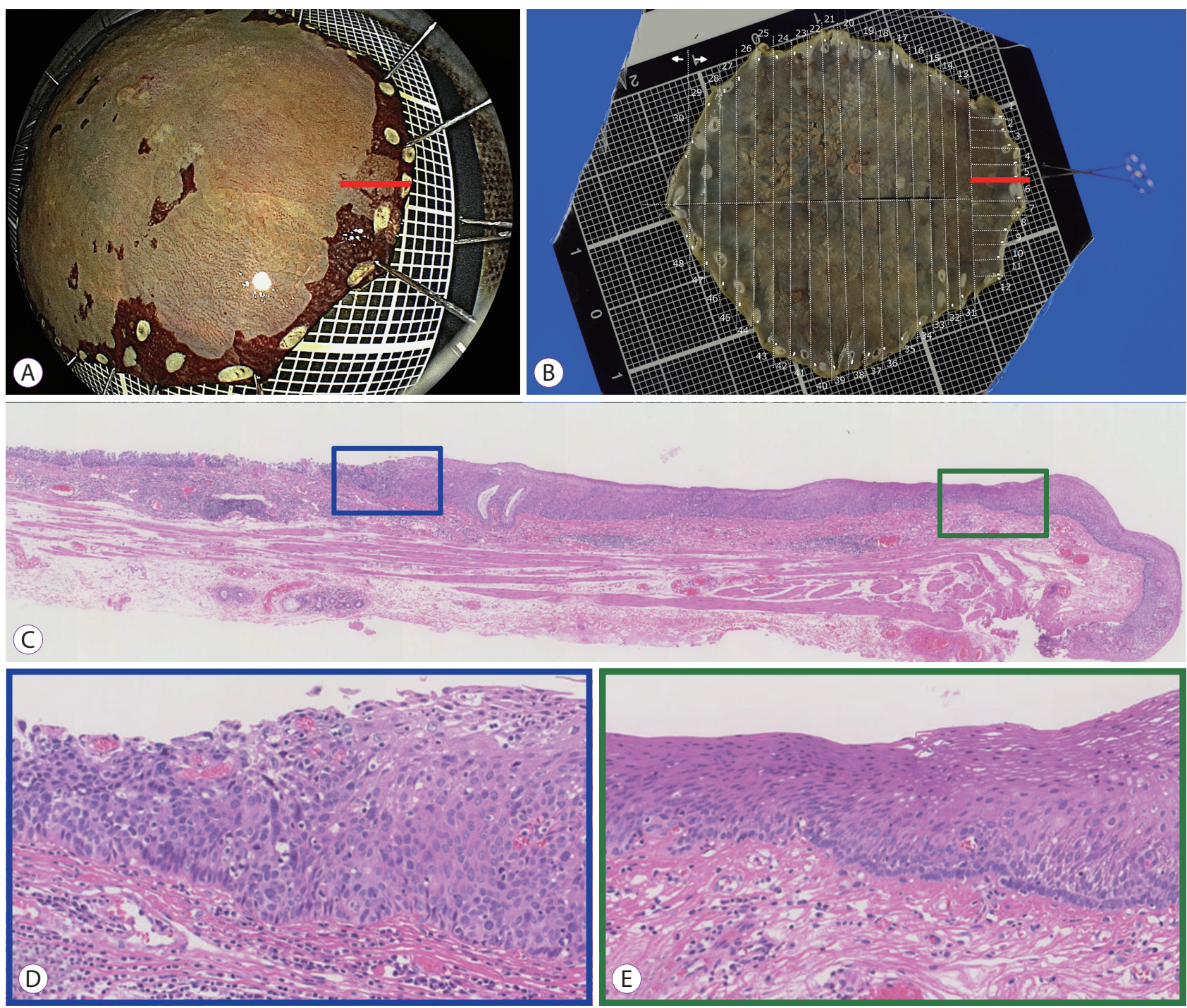

Fig. 2. Histological features of the resected specimen. (A, B) Endoscopically resected specimen—Red line indicates the area with different colors on linked color imaging and blue laser imaging. (C) Histopathological findings of the lesion indicated by the red line on image $A$ and $B$ (hematoxylin \& eosin, $\times 2.57$ ). (D) A magnified image shows the histopathological findings at the border between squamous cell carcinoma (left half) and intraepithelial neoplasia (right half) (hematoxylin \& eosin, $\times 8.17)$. (E) The magnified image shows the histopathological findings at the border between intraepithelial neoplasia (left half) and normal mucosa (right half) (hematoxylin \& eosin, $\times 8.17)$.

Table 1. Color Differences between the Malignant Region and Dysplastic Area (Calculated by the Commission Internationale de l'Eclairage Method)

\begin{tabular}{lcc}
\hline After iodine staining & $\begin{array}{c}\Delta \mathrm{E} \text { (Cancer and dysplastic regions color } \\
\text { difference) }\end{array}$ & $\begin{array}{c}\Delta \mathrm{E} \text { (Cancer and surrounding epithelium color } \\
\text { difference) }\end{array}$ \\
\hline WLI & 5.8 & 14.0 \\
BLI & 26.6 & 19.7 \\
LCI & 28.9 & 21.3 \\
\hline
\end{tabular}

By National Bureau of Standards unit the color difference evaluation: 0-0.5 Trace; 0.5-1.5 Slight; 1.5-3.0 Noticeable; 3.0-6.0 Appreciable; 6.0-12.0 Much, 12.0 and higher Very much.

BLI, blue laser imaging; LCI, linked color imaging; WLI, white light imaging. 
Therefore, appropriate knowledge of the utilization of these modalities is essential, facilitating the early detection of cancer as well as minimally invasive endoscopic treatment, in order to improve the quality of life of patients and reduce the mortality associated with esophageal squamous cell carcinoma.

Detection of superficial flat type esophageal squamous cell carcinoma is often difficult as only subtle changes are revealed in the mucosal surface. An accurate examination with iodine staining includes assessment of the pink-color sign, wherein color change is observed from an unstained area to a pink color three minutes after application of the iodine solution. The iodine solution adheres to the glycogen of the normal non-keratinized squamous epithelium of the esophagus, whereas a malignant tissue, dysplasia site, inflammatory site, or erosive mucosa lacks glycogen and remains unstained. ${ }^{13}$ However, accurate judgment of positive or negative pink-color sign may not be done by endoscopists owing to minimal difference between the pale pink stained area and non-pink color of the unstained area. In this patient, WLI demonstrated a pale pink color in the unstained area, which may have been difficult to judge as positive or negative.

Recently, image enhanced endoscopy was developed and the ability of this technique to detect esophageal lesions was reported to be greater than WLI, regardless of iodine staining. ${ }^{14}$ Esophageal squamous cell carcinoma appears as a silver metallic color on NBI after staining with iodine. ${ }^{15}$ However, multiple lesions or irregularly shaped areas may be visualized in iodine-unstained areas, thereby causing difficulty in recognition and precise ability to distinguish the cancer. ${ }^{10}$ In the present patient, BLI revealed green mucosa in the unstained area and interestingly LCI showed purple mucosa, both of which allowed the endoscopist to identify an abnormal lesion compared with the use of WLI. Visualization of partially non-purple and non-green portions in the unstained area using LCI and BLI, respectively was unanticipated. Endoscopic submucosal dissection specimens revealed that the purple and green areas were squamous cell carcinoma whereas the non-purple and non-green areas were squamous intraepithelial neoplasia. These findings may be useful to identify squamous cell carcinoma among multiple iodine-unstained lesions that can often be observed in patients with risk factors such as smoking and intake of alcohol.

Previous studies have reported the usefulness of color differences in the identification of malignant lesions. ${ }^{16,17}$ In the present case; the color difference was calculated in all three modalities including WLI, LCI, and BLI before and after iodine staining and comparison of the malignant lesion was performed with the surrounding mucosa as well as the dysplastic regions (Table 1). These results showed that the color differences between esophageal squamous cell carcinoma and dysplasia as well as those between esophageal squamous cell carcinoma and normal mucosa were greatest using LCI, which explains the easier recognition by visual inspection. The reason for greater color difference on LCI remains unclear; however, it may be hypothesized that the presence or absence of normal squamous cells in the epithelium may influence the color visualized. Malignant cells are evident in the entire layer of esophageal epithelium in carcinoma but not in areas of intraepithelial neoplasia that include normal squamous cells in the superficial layer. LCI and BLI have high emission intensity of short wavelengths that reflect from the mucosal surface. ${ }^{18}$ This histological difference may influence the spectral reflectance of both epithelia even after iodine staining, which results in different colors.

In conclusion, LCI and BLI combined with iodine staining produced characteristic images that overcame the pink-color sign, reflecting histological features of a flat esophageal neoplasm. This new approach is useful to evaluate and diagnose early flat squamous cell neoplasms in detail, and deserves further study.

\section{Conflicts of Interest}

Hiroyuki Osawa and Hironori Yamamoto received honoraria from Fujifilm Corporation and Fujifilm Medical Corporation. Other authors have no conflicts of interest.

\section{REFERENCES}

1. Wang J, Liu F, Gao H, et al. The symptom-to-treatment delay and stage at the time of treatment in cancer of esophagus. Jpn J Clin Oncol 2008;38:87-91.

2. Ishihara R, Takeuchi Y, Chatani R, et al. Prospective evaluation of narrow-band imaging endoscopy for screening of esophageal squamous mucosal high-grade neoplasia in experienced and less experienced endoscopists. Dis Esophagus 2010;23:480-486.

3. Muto M, Minashi K, Yano T, et al. Early detection of superficial squamous cell carcinoma in the head and neck region and esophagus by narrow band imaging: a multicenter randomized controlled trial. J Clin Oncol 2010;28:1566-1572.

4. Yokoyama A, Ichimasa K, Ishiguro T, et al. Is it proper to use non-magnified narrow-band imaging for esophageal neoplasia screening? Japanese single-center, prospective study. Dig Endosc 2012;24:412-418.

5. Ebi M, Shimura T, Yamada T, et al. Multicenter, prospective trial of white-light imaging alone versus white-light imaging followed by magnifying endoscopy with narrow-band imaging for the real-time imaging and diagnosis of invasion depth in superficial esophageal squamous cell carcinoma. Gastrointest Endosc 2015;81:1355-1361.e2.

6. Tomie A, Dohi O, Yagi N, et al. Blue laser imaging-bright improves endoscopic recognition of superficial esophageal squamous cell carcinoma. Gastroenterol Res Pract 2016;2016:6140854.

7. Diao W, Huang X, Shen L, Zeng Z. Diagnostic ability of blue laser imaging combined with magnifying endoscopy for early esophageal cancer. Dig Liver Dis 2018;50:1035-1040.

8. Osawa H, Yamamoto H, Miura Y, et al. Blue laser imaging provides excellent endoscopic images of upper gastrointestinal lesions. Video Journal and Encyclopedia of GI Endoscopy 2014;1:607-610. 
9. Ishihara R, Yamada T, Iishi H, et al. Quantitative analysis of the color change after iodine staining for diagnosing esophageal high-grade intraepithelial neoplasia and invasive cancer. Gastrointest Endosc 2009;69:213-218.

10. Goda K, Dobashi A, Yoshimura N, et al. Narrow-band imaging magnifying endoscopy versus Lugol chromoendoscopy with pink-color sign assessment in the diagnosis of superficial esophageal squamous neoplasms: a randomised noninferiority trial. Gastroenterol Res Pract 2015;2015:639462.

11. Oyama T, Inoue H, Arima M, et al. Prediction of the invasion depth of superficial squamous cell carcinoma based on microvessel morphology: magnifying endoscopic classification of the Japan Esophageal Society. Esophagus 2017;14:105-112.

12. Kuehni RG. Color-tolerance data and the tentative CIE 1976 L a b formula. J Opt Soc Am 1976;66:497-500.

13. Shimizu Y, Omori T, Yokoyama A, et al. Endoscopic diagnosis of early squamous neoplasia of the esophagus with iodine staining: high-grade intra-epithelial neoplasia turns pink within a few minutes. J Gastroen- terol Hepatol 2008;23:546-550.

14. Nagami $Y$, Tominaga $K$, Machida $H$, et al. Usefulness of non-magnifying narrow-band imaging in screening of early esophageal squamous cell carcinoma: a prospective comparative study using propensity score matching. Am J Gastroenterol 2014;109:845-854.

15. Maselli $\mathrm{R}$, Inoue $\mathrm{H}$, Ikeda $\mathrm{H}$, et al. The metallic silver sign with narrow-band imaging: a new endoscopic predictor for pharyngeal and esophageal neoplasia. Gastrointest Endosc 2013;78:551-553.

16. Osawa H, Yamamoto H, Miura Y, et al. Diagnosis of depressed-type early gastric cancer using small-caliber endoscopy with flexible spectral imaging color enhancement. Dig Endosc 2012;24:231-236.

17. Fukuda H, Miura Y, Osawa H, et al. Linked color imaging can enhance recognition of early gastric cancer by high color contrast to surrounding gastric intestinal metaplasia. J Gastroenterol 2018 Oct 5 [Epub]. https:// doi.org/10.1007/s00535-018-1515-6.

18. Osawa H, Miura Y, Takezawa T, et al. Linked color imaging and blue laser imaging for upper gastrointestinal screening. Clin Endosc 2018;51:513-526. 